\title{
Model of data center temperature monitoring system with the use of open source hardware
}

\author{
Dalibor Dobrilović*, Željko Stojanov*, Zlatko Čović**, Janos Simon** and Nikola Petrov* \\ *University of Novi Sad / Technical Faculty "Mihajlo Pupin", Zrenjanin, Serbia \\ ${ }^{* *}$ Subotica Tech, Department of Informatics, Subotica, Serbia \\ dalibor.dobrilovic@uns.ac.rs, zeljko.stojanov@uns.ac.rs, chole@vts.su.ac.rs, simon@vts.su.ac.rs, \\ nikola.petrov@tfzr.rs
}

\begin{abstract}
This paper puts in the focus two important segments of information and communication technology. The first segment is monitoring of data centers and the second one is usage of open source hardware. The reason for combining these two segments is effort to explore the efficiency of open source hardware platforms in these environments. The advantages of open source hardware platform as a tool for rapid prototype development and combination of variety of technologies were taken as the main starting point for this research. This paper describes the model for data center temperature monitoring with the usage of open source hardware. The prototype system is partially implemented in accordance with the presented model.
\end{abstract}

\section{INTRODUCTION}

The importance of monitoring data centers has its significant place. The focus in this research is made on monitoring temperature parameters of data centers. The model presented here is focused on temperature, but it is not limited to this. Temperature is taken because it is by far the most present measurement parameter in the data centers. Reasons for this approach is that temperature could be monitored on network devices, UPS systems and computers (e.g. on CPUs as well as on GPUs). Network devices incorporated in focused data center have variety of built-in sensors mainly for reading temperature. This ability is combined with open source hardware in order to create temperature monitoring system for tracking temperature changes inside the equipment, inside the rack and at various places in the data center room.

The paper is organized as follows: after the introduction, the brief overview of datacenter monitoring systems is presented. Next, the data center configuration and its equipment are presented in the third section. After the equipment, the data center temperature monitoring model is presented. The partial implementation of the model and its validation is given in the fifth section. At the end, the discussion and deep analyses of further work are presented.

\section{DATA CENTER TEMPERATURE Monitoring SYSTEMS}

Actuality of the data center monitoring systems is shown in number of different solutions using variety of technologies $[1,2]$. The solutions differ depending on the size of the data centers and their configuration. The usages of robots are explained in $[3,12,13]$, while the integration of Wireless Sensor Network (WSN) technologies has become more evident in recent years $[4,5,6,20]$. The interesting examples of monitoring microclimate conditions in green house are given in $[14,15]$.

The novelty of the approach presented in this paper is in the effort made to efficiently combine open-source platform with the built-in sensors in the devices deployed in the data center. Although, there is a lot of commercial data center monitoring systems, this approach gives flexible, low-cost platform, which can be easily changed in order to enable development of the effective, optimal and stable data center monitoring systems. The strength of the proposed model is justified in addition with the planned expansions described in the Conclusion and further works section.

\section{DATA CENTER}

The data center which is in the focus for building this model is based on [7]. Its purpose and configuration are explained in more details in same references. In brief, the equipment around which is built datacenter is financed by project MIS ETC 1379 "Cross-border access infrastructure to high-level education through web-casts (EduWebCast)" and it is financed by Romania - Republic of Serbia IPA Cross-border Cooperation Programme, financed by the European Union under the Instrument for Pre-accession Assistance (IPA) and co-financed by the partner states in the programme. The system is build to enable efficient cross border educational web casting between two universities from two bordering countries, Romania and Serbia. Hardware of the data center is presented in Fig. 1 Technologies and platforms used for the system are explained in the following text.

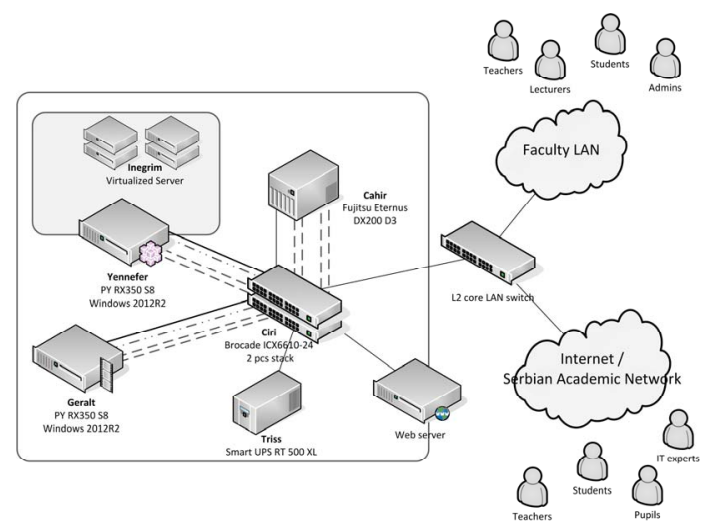

Figure 1. Components of the Serbian side EduWebCast system 


\section{A. Data Center hardware}

The system is installed as an integrated part of the academic LAN behind the institution firewall. It is connected via core L3 switch with the Internet. Application server, hosting the web application for the site and server used as video server are also connected to the switch. Video server has one public and one private IP address. The rest of the system is in the separate segment of LAN network. The central part of this segment is core switch for LAN and SAN (Storage Area Network) connections.

This core L3 switch is composed of two stackable switches connected via stacking 40GbE QSFP port. Together, those switches have 48 1Gbps ports for connecting the computers and other network equipment in the segment, $810 \mathrm{Gbps}$ ports for enabling fiber optics connection between 2 servers and storage device for data transfer using iSCSI technology (red dashed lines of Fig. 3 ). Each server has its connection over two controller cards. The switch has two additional 10Gbps ports for connecting the switch to the network [7].
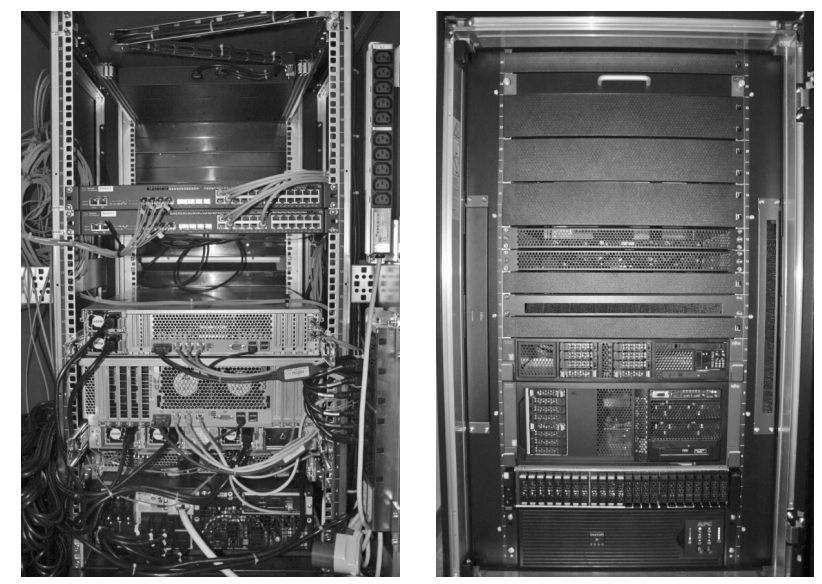

Figure 2. Front and rear view of EduWebCast system rack

The storage device has 12 SAS disks in the current configuration with the capacity of 600GB giving in total 7.2 TB of storage capacity. Ten disks are in operation mode and two are in spare mode, acting as reserve disks. The number of disks is not limited to 12 and the storage system is easy to expand in the case of need [7].

Disk RAID Level is set to High Performance (RAID1+0) with total operating capacity of 2.67 TB. One part of the disk has capacity of 900.00 GB $(943718400.00$ $\mathrm{KB})$ and this part is used by the application server. The other part of the disk has capacity of 1.79 TB (1918894080.00 KB) and this disk is used for video server. The video server part of disk space is used for storing its operating system, data and video content.

Video server is a computer with 64GB of RAM, two Xeon E5-2697 v2 12C/24T 2.7GHz CPUs and two GPUs TeslaK20X. Video server hosts the video streaming server application. The system is secured with $5000 \mathrm{kVA}$ UPS system for continuous power supply [7].

\section{B. Device built-in sensors}

In order to make the profile of capture data clear, in this subsection, types of sensors implemented in data center network devices and servers are described. The profile of sensors for two stacked Brocade ICX 6610 switches is shown with the Brocade CLI command show chassis. In Listing 1 it is shown that this command gives number of temperature parameters given as follows: fan, MAC, CPU, Sensor A, Sensor B, Sensor C, Sensor D and Stacking card.

The MAC temperature is used as a reference temperature because it is only displayed in the web administration page of the device. It is important to point that two stacked devices are placed in the rack as it is show in Fig. 2 and the lower stacked Brocade switch is giving higher temperature measures. This is expected because the lower switch is located in the center of the switch and it is practically surrounded with the other equipment. Considering that there are two stacked Brocade switches, total of 16 temperature sensor readings are monitored [16].

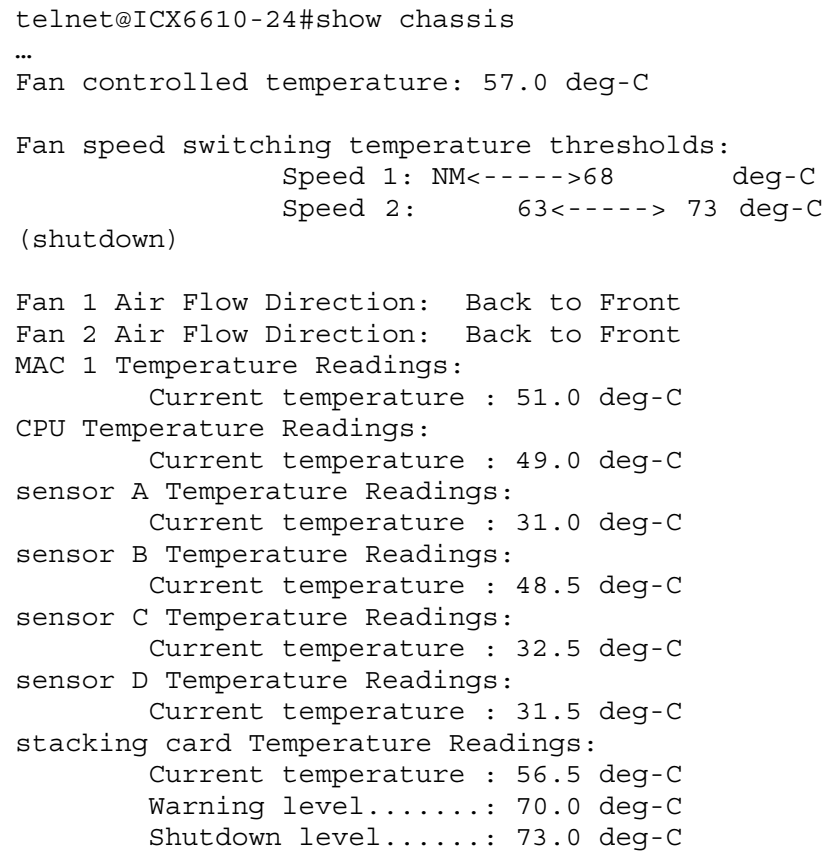

Listing 1. Output of show chassis command at Brocade ICX 6610 switch

The next data sensors are implemented in UPS system. The UPS is APC Online UPS S2 5kVA / 3,5kW R/T. Two commands showing sensors readings are given in Listing 2. One reading relates to internal temperature and another one is related to power consumption data [17].

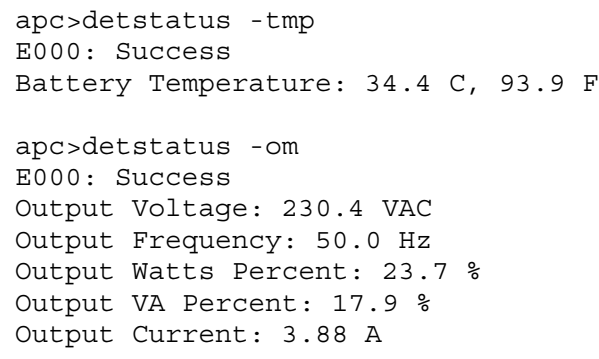

Listing 2. Output of detstatus -tmp and detstatus -om command at APC Online UPS 
The third device with sensors is external data storage Fujitsu Eternus DX200. Four temperature sensor readings are monitored from this device using CLI command show enclosure-status -type ce. The output of the command is given in Listing 3 [18].

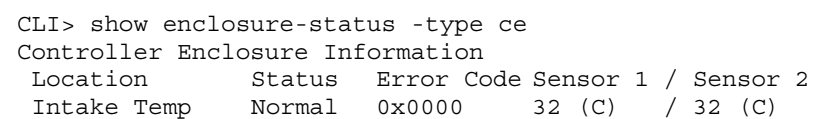

Listing 3. Output of show enclosure-status at Fujitsu Eternus DX200 storage

The next sensors which can be read are the temperature sensors from two servers. First server has two Intel Xeon E5-2697v2 CPUs with 12 cores. Each processor can give 12 CPU temperature readings giving in total 24 sensors. Second server has two Xeon E5-2697 v2 with 10 cores, giving in total 20 additional temperature sensors. All readings of the CPU temperatures are not implemented in the current prototype, and are made and logged with OpenHardwareMonitor tools for testing and experimental purpose.

The GPU temperature readings can be obtained with nvidia-smi tool. The example is shown in Listing 4, as well as an example with filtered data in Listing 5.

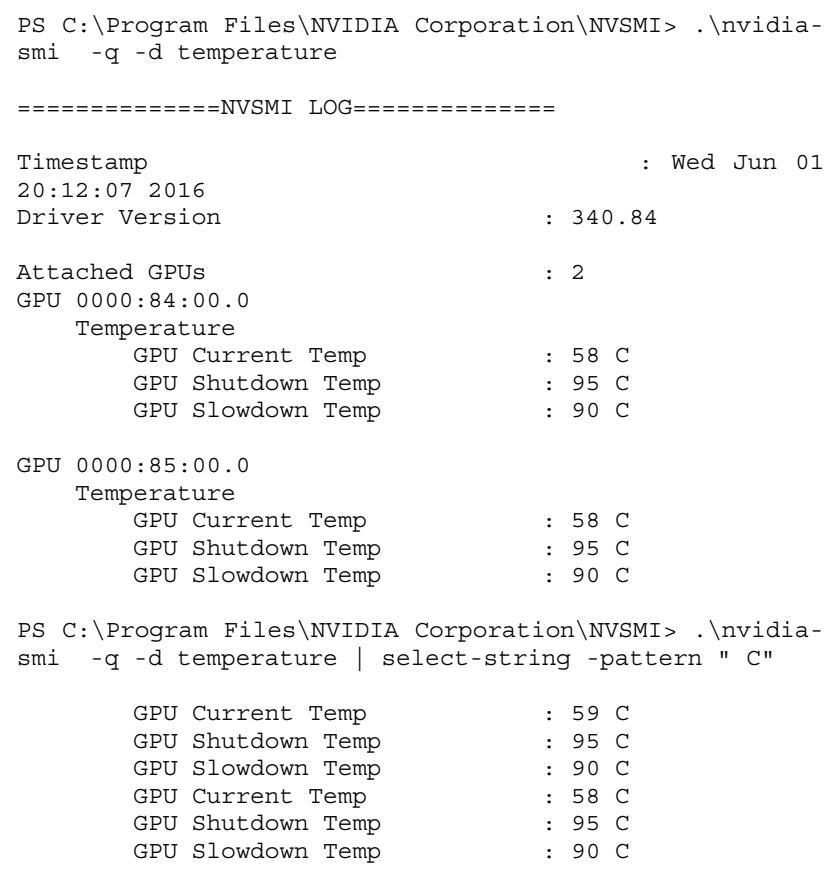

PS C: \Program Files $\backslash$ NVIDIA Corporation \NVSMI> . \nvidiasmi -q -d temperature | select-string -pattern " C"

$\begin{array}{ll}\text { GPU Current Temp } & : 59 \mathrm{C} \\ \text { GPU Shutdown Temp } & : 95 \mathrm{C} \\ \text { GPU Slowdown Temp } & : 90 \mathrm{C} \\ \text { GPU Current Temp } & : 58 \mathrm{C} \\ \text { GPU Shutdown Temp } & : 95 \mathrm{C} \\ \text { GPU Slowdown Temp } & : 90 \mathrm{C}\end{array}$

Listing 4. Output of nvidia-smi command at Windows 2012R power shell

PS C: $\backslash$ Program Files $\backslash$ NVIDIA Corporation $\backslash$ NVSMI $>$. \nvidiasmi -q -d temperature | select-string -pattern " C" | select-str

ing -pattern "Current"

$$
\begin{array}{ll}
\text { GPU Current Temp } & : 59 \mathrm{C} \\
\text { GPU Current Temp } & : 58 \mathrm{C}
\end{array}
$$

Listing 5. Output of nvidia-smi command at Windows 2012R power shell with filtered output
Two GPU Tesla K20X temperature readings provide totally $46 \mathrm{CPU} / \mathrm{GPU}$ temperatures for monitoring. The GPU readings are not implemented in the current prototype in this phase of the research.

OpenHardwareMonitor is also capable for monitoring CPU power, cores and DRAM consumption, hard disk and memory load. All these data can be added to monitoring system eventually.

\section{Open source hardware wireless sensor network}

In order to add sensor readings outside the rack to this system, three wireless sensor network stations based on open source hardware microcontroller board Arduino/Genuino UNO Rev3 and ZigBee communication modules are added. ZigBee communication modules are well-known Digi International XBee ${ }^{\circledR}$ Series 2 modules. These modules are already used for research in our institution [9, 10] and for wider purposes [11]. Three WSN nodes are configured as ZigBee routers and one ZigBee coordinator module (4) is connected via USB dongle to the second server in data center. This server is used to collect WSN data station readings.

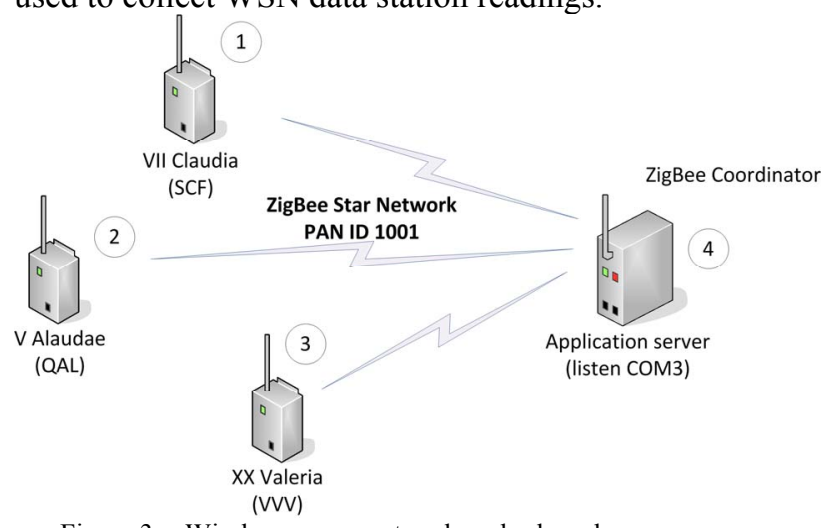

Figure 3. Wireless sensor network nodes based on open source hardware

Hardware configurations of three stations are presented in Table I. The reason for choosing these configurations is that they allow solderless and easy to configure and to program platforms. Platform programming is made with Arduino IDE, and the most important thing in these applications is to read sensor values, to do preprocessing if it is necessary, and to send sensor values using ZigBee communication modules.

In order to acquire temperature outside of the equipment three sensor stations are placed on different locations in the data center. Station QAL (2) is located inside of the rack, on the top of higher Brocade switch. Station VVV (3) is located on the top of the rack. And third station SCF (1) is located on the $2 \mathrm{~m}$ distance from the rack in the bottom part of the room window. The configurations of the sensor stations show that they are using mainly temperature sensors, and the most different one is light sensor implemented in SCF station. Since that station is located close to the window it is used to measure amount of light entering the data center room.

The configuration of sensor stations in this case is focused on temperature sensors, so the multiple temperature sensors are used on single station in order to control the accuracy of the sensors, since the low-cost 
versions are used. The usage of other sensor, particularly suitable for the usage in data center monitoring will be discussed in the section with the further work.

TABLE I. CONFIGURATION OF WIRELESS SENSOR STATIONS

\begin{tabular}{|c|c|c|c|c|}
\hline Station name & Station ID & Components & Sensors & Types \\
\hline \multirow[b]{2}{*}{ VII Claudia } & \multirow[b]{2}{*}{$\mathrm{SCF}$} & \multirow{2}{*}{$\begin{array}{l}\text { Arduino Uno R3 } \\
\text { Freaduino Sensor Shield v1.4 } \\
\text { XBee series 2 } \\
\text { Duck antenna 2dBi }\end{array}$} & DHT11 & $\begin{array}{l}\text { Temperature } \\
\text { Humidity }\end{array}$ \\
\hline & & & BH1750FVI & Light \\
\hline \multirow[b]{2}{*}{ V Alaudae } & \multirow[b]{2}{*}{ QAL } & \multirow{2}{*}{$\begin{array}{l}\text { Arduino Uno R3 } \\
\text { Freaduino Sensor Shield v1.4 } \\
\text { XBee series 2 } \\
\text { Duck antena 2dBi }\end{array}$} & $\begin{array}{l}\text { Grove } \\
\text { Temp }\end{array}$ & Temperature \\
\hline & & & BMP085 & $\begin{array}{l}\text { Temperature } \\
\text { Barrometric } \\
\text { preassure }\end{array}$ \\
\hline \multirow[t]{2}{*}{ XX Valeria } & \multirow[t]{2}{*}{ VVV } & \multirow{2}{*}{\begin{tabular}{|l|} 
Arduino Uno R3 \\
DFDuino I/O Expansion Shield \\
V5.0 \\
XBee series 2 \\
Duck antenna 2dBi \\
\end{tabular}} & BMP085 & $\begin{array}{l}\text { Temperature } \\
\text { Barrometric } \\
\text { preassure }\end{array}$ \\
\hline & & & DS18B20 & Temperature \\
\hline
\end{tabular}

IV. MODEL OF DATA CENTER TEMPERATURE MONITORING SYSTEM

The model of the system for monitoring data center temperature with its main components is show in Fig. 4. The light gray modules are made as a prototype in order to validate this model and to make good basis for the future work. The dark gray modules on the figure are modules that are used as third party software. All modules are in this first phase of development, and they are built to run separately and to log sensor data in CSV files. The previously built application is used for parsing $\log$ files and for generating result shown in next section.

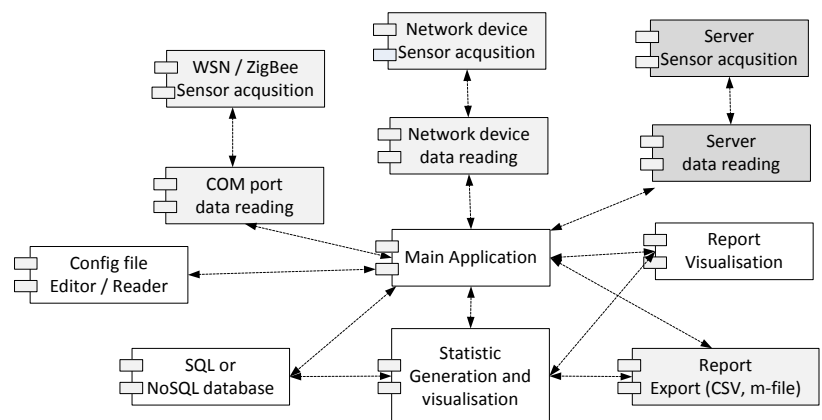

Figure 4. Component diagram of the data center monitoring system expanded with open source hardware

\section{THE RESULTS}

The results of the device sensor temperature readings are given in Fig.5. The readings are from up and down brocade switch MAC1 sensor, UPS battery temperature sensor and two SFP temperatures from external storage.

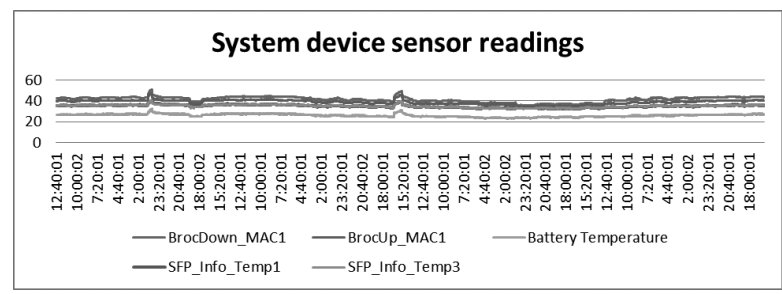

Figure 5. Device sensor readings

In Fig. 6 are shown results of following sensors: two temperature sensors (BMP085 and DS18B20) from VVV wireless sensor station located on the top of the rack (bottom two curves); two temperature sensors (Grove temp and BMP085) from QAL wireless sensor station located inside the rack (top two curves); and one temperature sensor (DHT11) from SCF wireless sensor station located close to the window (middle curve).

The usage of wireless ZigBee based sensor station proves to be efficient. The sensor nodes operated without any significant problems during three months period of testing and experimenting.

The collected data appear to be useful for monitoring dependencies between ambient and inner device temperature. Also, the potential data analyses can lead to optimization of the cooling temperature of air conditioner devices in order to make energy savings. The data analytics are not implemented in the prototype yet, but are certainly planned as statistic and report generation modules.

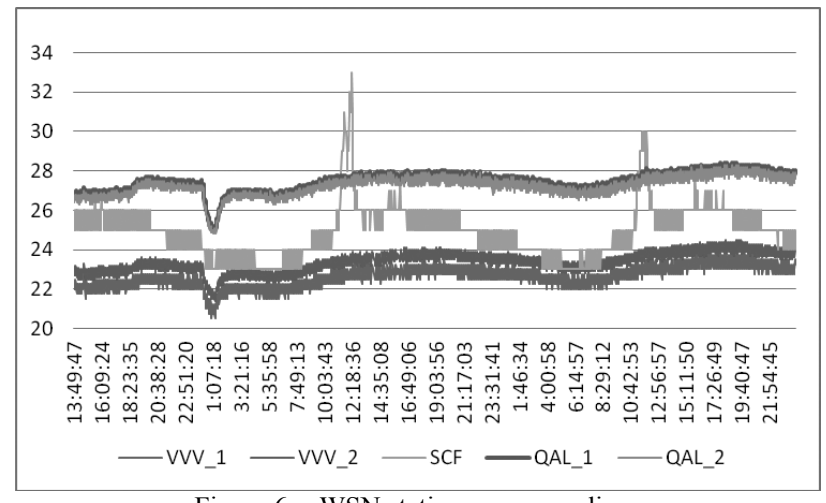

Figure 6. WSN station sensor readings

The usage of wireless sensor nodes is justified with their easy and solderless assembling. The configuration of sensor nodes can be easily changed with adding the other type of sensors. For data center, the sensors for dust, sound, and current consumption can be interested. In addition, the addition of PIR motion sensors and web cameras can make wider monitoring system usage by adding video surveillance features as well [20]. The integration of the platform with the cloud systems is also possible [21].

\section{CONCLUSION AND FURTHER WORK}

In this paper the model of data center monitoring system is presented. The monitoring system is in this stage mainly focused on the temperature monitoring. The 26 sensors from network devices (LAN/SAN switch, external storage and UPS) are included in the model. The additional $46 \mathrm{CPU} / \mathrm{GPU}$ sensors from two servers, as well as 6 sensors from ZigBee wireless sensor nodes are added. This gives in total of 78 monitoring sensors. Only part of the model is built as a prototype in the current phase. This part is data acquisition from device and wireless node sensors. The data logging part is implemented as well.

The system is put in experimental work for 3 months. The special attention is devoted to monitoring the work of wireless sensor stations based on Arduino/Genuino UNO Rev 3, as a rapid development and prototyping platform. 
The Arduino/Genuino nodes, as well as XBee ZigBee communication modules proved to be efficient and reliable during the testing period.

The system can be expanded. In the first stage it can be expanded with sound, dust, light and current consumption sensors. In the second stage they can be expanded with video surveillance features, motion detectors and web cams. Finally, they can be expended with IoT (Internet of Things) features and Cloud systems, to be in compliance with modern trends, as well as to allow trusted shared data center monitoring, from trusted location and persons. Software modules for data analytics and reporting are also planned to be developed for extending processing and reporting capabilities of the system.

\section{ACKNOWLEDGMENT}

This research is supported by Ministry of Education and Science of the Republic of Serbia under the project number TR32044 "The development of software tools for business process analysis and improvement”, 2011-2016.

\section{REFERENCES}

[1] Babak Lajevardi, Karl R. Haapala, Joseph F. Junker, Real-time monitoring and evaluation of energy efficiency and thermal management of data centers, Journal of Manufacturing Systems, Volume 37, Part 2, October 2015, Pages 511-516, ISSN 02786125, http://dx.doi.org/10.1016/j.jmsy.2014.06.008.

[2] Chengwei Wang, Karsten Schwan, Vanish Talwar, Greg Eisenhauer, Liting $\mathrm{Hu}$, and Matthew Wolf. 2011. A flexible architecture integrating monitoring and analytics for managing large-scale data centers. In Proceedings of the 8th ACM international conference on Autonomic computing (ICAC '11). ACM, New York, NY, USA, 141-150. DOI=http://dx.doi.org/10.1145/1998582.1998605

[3] C. Mansley et al., "Robotic mapping and monitoring of data centers," Robotics and Automation (ICRA), 2011 IEEE International Conference on, Shanghai, 2011, pp. 5905-5910. doi: 10.1109/ICRA.2011.5980554

[4] E. A. Kadir, S. M. Shamsuddin, S. Hasan and S. L. Rosa, "Wireless monitoring for big data center server room and equipments," 2015 International Conference on Science in Information Technology (ICSITech), Yogyakarta, 2015, pp. 187191. doi: 10.1109/ICSITech.2015.7407801

[5] S. K. Vuppala, A. Ghosh, K. A. Patil and K. Padmanabh, "A Scalable WSN Based Data Center Monitoring Solution with Probabilistic Event Prediction," 2012 IEEE 26th International Conference on Advanced Information Networking and Applications, Fukuoka, 2012, pp. 446-453.

[6] R. Khanna and H. Liu, "Machine learning approach to data center monitoring using wireless sensor networks," Global Communications Conference (GLOBECOM), 2012 IEEE,
Anaheim, CA, 2012, pp. 689-694. doi: 10.1109/GLOCOM.2012.6503193

[7] M. Marcu, S. Fuicu, D. Dobrilovic, M. Popa and B. Odadzic, Cross-border infrastructure for educational webcasting, International conference on Applied Internet and Information Technologies AIIT 2015, Zrenjanin, Serbia, October 23, 2015.

[8] Open Hardware Monitor official site, http://openhardwaremonitor. org/, retrieved on May 2016.

[9] Dalibor Dobrilovic, Zeljko Stojanov, Vladimir Brtka, Zlatko Covic and Nemanja Bilinac. Software application for analyzing ZigBee network performance designed for teaching and testing Wireless Sensor Networks. In Proceedings of the IEEE 12th International Symposium on Intelligent Systems and Informatics (SISY 2014), pp. 72-77. September 11-13 2014. Subotica, Serbia.

[10] D. Dobrilovic, Z. Stojanov, B. Odadzic, "Teaching Application Development for RFID/ZigBee Networks using Open Source Hardware", Proceeding of IEEE 10th International Symposium on Telecommunications BIHTEL 2014, October 27-29, Sarajevo, Bosnia and Herzegovina, 2014.

[11] Robert Faludi, Building Wireless Sensor Networks, O'Reilly Media, Inc., USA, 2011.

[12] Simon János, "Concepts of the Internet of Things from the Aspect of the Autonomous Mobile Robots", Interdisciplinary Description of Complex Systems Vol.13 No.1, pp. 34-40, 2015.

[13] Simon János, Zlatko Covic, "Data Management of the Automomous Mobile Devices and Internet of Things", ANNALS of Faculty Engineering Hunedoara - International Journal of Engineering Vol. XIII, No. 3, pp. 265-268, 2015.

[14] Goran Martinović, Simon János, "Greenhouse Microclimatic Environment Controlled by a Mobile Measuring Station", Journal of the Royal Netherlands Society for Agricultural Sciences, Vol. 70, No. 1, pp. 61-70, 2014.

[15] Simon János, "Optimal Microclimatic Control Strategy Using Wireless Sensor Network and Mobile Robot", Acta Agriculturae Serbica Vol. XVIII, No. 36, pp. 3-12, 2013.

[16] FastIron Ethernet Switch - Administration Guide, Supporting FastIron Software Release 08.0.30, 53-1003625-01, Brocade Communications Systems, Inc., 31 March 2015.

[17] Command Line Interface Guide - UPS Network Management Card 2, AP9630, AP9631, AP9635, 990-4879B-001, 10/2015, (C) 2015 APC by Schneider Electric.

[18] ETERNUS CLI User's Guide, P2X0-1030-09ENZ0, FUJITSU LIMITED, August 2015.

[19] D. Dobrilovic, B. Odadzic, Z. Stojanov and V. Sinik, "Testing Zigbee RF module applicability for usage in temperature monitoring systems," Telecommunications Forum Telfor (TELFOR), 2014 22nd, Belgrade, 2014, pp. 415-418.doi: 10.1109/TELFOR.2014.7034436

[20] Milan Malić, Dalibor Dobrilović, Ivana Petrov, Example of IoT platform usage for wireless video surveillance with support of NoSQL and cloud systems, In Proceedings of 6th International Conference on Applied Internet and Information Technologies AIIT2016, 03-04 June, Bitola, Macedonia, 2106.

[21] Nikola Petrov, Dalibor Dobrilovic, Mila Kavalić, Sanja Stanisavljev, Examples of Raspberry Pi usage in Internet of Things, In Proceedings of 6th International Conference on Applied Internet and Information Technologies AIIT2016, 03-04 June, Bitola, Macedonia, 2106. 